\title{
Reflexão sobre a organização do trabalho de Enfermagem no banco de leite: cuidado compartilhado e multiprofissional
}

\author{
Reflection on the organization of Nursing work in the milk bank: shared and multidisciplinary care \\ Reflexión sobre la organización del trabajo de Enfermería en el banco de leche: cuidado compartido y \\ multiprofesional
}

Giovanna Rosario Soanno Marchiori ${ }^{1}$ (D) Valdecyr Herdy Alves ${ }^{2}$ (1)

Diego Pereira Rodrigues ${ }^{3}$ (D) Bianca Dargam Gomes Vieira² (1)

Audrey Vidal Pereira ${ }^{2}$ (1)

Tatiana do Socorro dos Santos

Calandrini ${ }^{4}$ (D)

1. Universidade Federal Fluminense,

Programa Acadêmico em Ciências do Cuidado em Saúde. Niterói, RJ, Brasil.

2. Universidade Federal Fluminense, Escola de Enfermagem Aurora de Afonso Costa. Niterói, RJ, Brasil.

3. Universidade Federal do Pará, Centro de Ciências da Saúde. Belém, PA, Brasil.

4. Universidade Federal do Amapá, Faculdade de Enfermagem. Macapá, AP, Brasil.
Autor correspondente:

Giovanna Rosario Soanno Marchiori. E-mail: giovannasoanno@gmail.com

Recebido em 26/05/2021.

Aprovado em 31/10/2021.

\section{Resumo}

Objetivo: refletir sobre a organização do trabalho de Enfermagem no Banco de Leite Humano a partir das ações de cuidado compartilhado com a equipe multiprofissional. Método: trata-se de um estudo teórico-reflexivo com base nos conceitos de campo e habitus de Pierre Bourdieu de modo a compreender o modus operandi dos seus agentes no campo e no subcampo da saúde. Resultados: evidenciou-se que o Processo de Enfermagem faz parte dos procedimentos de cuidado em saúde nos diferentes espaços de atuação, todavia, é importante pontuar que há uma interdependência dos serviços prestados. Percebem-se a integralidade da proposta e a organização do trabalho do BLH a partir de uma equipe multiprofissional. Para a Enfermagem, o habitus está posto desde a formação inicial dos seus profissionais, pois há o reconhecimento do saber próprio do campo e que as ações são pautadas em conhecimentos teórico-científicos. Conclusão e implicações para a prática: o cuidado compartilhado é reconhecido como importante fator de equalização da assistência e demais serviços realizados no campo do BLH. Ao mobilizar os conceitos de Pierre Bourdieu, é possível inferir o habitus nos modos de cuidar, considerando-se, ainda, aquele específico construído ao longo da história da Enfermagem.

Palavras-chave: Aleitamento materno; Assistência à saúde; Banco de leite humano; Equipe multiprofissional; Processo de enfermagem.

\section{Abstract}

Objective: reflect on the organization of nursing work in the Human Milk Bank based on shared care actions with the multiprofessional team. Method: this is a theoretical-reflexive study based on Pierre Bourdieu's concepts of field and habitus in order to understand the modus operandi of its agents in the field and subfield of health. Results: it was evidenced that the Nursing Process is part of the health care procedures in the different performance spaces, however, it is important to point out that there is an interdependence of the services provided. The integrality of the proposal and the organization of the work of the HMB are perceived from a multi-professional team. For nursing, the habitus is set from the initial training of its professionals, since there is recognition of the knowledge of the field and that the actions are based on theoretical and scientific knowledge. Conclusion and implications for practice: shared care is recognized as an important factor in equalizing care and other services performed in the field of HMB. By mobilizing the concepts of Pierre Bourdieu, it is possible to infer the habitus in the modes of care, considering also the specific habitus built throughout the history of Nursing.

Keywords: Breastfeeding; Delivery of health care; Human milk bank; Multi-professional team; Nursing process.

\section{Resumen}

Objetivo: reflexionar sobre la organización del trabajo de Enfermería en el Banco de Leche Materna, a partir de acciones de cuidado compartido con el equipo multiprofesional. Método: es un estudio teórico-reflexivo basado en los conceptos de campo y habitus de Pierre Bourdieu, con el fin de comprender el modus operandi de sus agentes en el campo y en el subcampo de la salud. Resultados: mostraron que el Proceso de Enfermería es parte de los procedimientos de atención en salud en diferentes áreas de actuación, sin embargo, es importante señalar que existe una interdependencia de los servicios brindados. La integridad de la propuesta y la organización del trabajo del BLM se puede ver desde un equipo multiprofesional. Para la Enfermería, el habitus se establece a partir de la formación inicial de sus profesionales, pues se reconoce el conocimiento del campo y que las acciones se basan en conocimientos teórico-científicos. Conclusión e implicaciones para la práctica: la atención compartida se reconoce como un factor importante en la igualación de la atención y otros servicios realizados en el campo del BLM. Mediante la movilización de los conceptos de Pierre Bourdieu, es posible inferir el habitus en los caminos de cuidado, teniendo en cuenta incluso el específico construido a lo largo de la historia de la Enfermería.

Palabras clave: Amamantamiento; Prestación de atención de salud; Banco de leche materna; Equipo multiprofesional; Proceso de enfermería 


\section{INTRODUÇÃO}

A presença da Enfermagem no Banco de Leite Humano (BLH) traz uma prática de assistência em um campo de atuação na promoção, proteção e apoio ao aleitamento materno, um cuidado que vai além desse espaço, alcançando, muitas vezes, outros setores da maternidade e do hospital, chegando até o domicílio dessas famílias que ali são assistidas. Esse espaço necessita que os profissionais de saúde que ali atuam estejam alicerçados na pesquisa, no saber técnico e científico, entendendo que essas dimensões são interdependentes e inter-relacionadas com o atendimento empático e visando às respostas humanas em todas as suas dimensões ${ }^{1}$.

No Brasil, os BLH desenvolvem suas ações do trabalho em rede, modelo que se configura no desenvolvimento de ações estratégicas da Política Nacional de Aleitamento Materno, sendo assim denominada Rede Brasileira de Bancos de Leite Humano (rBLH-BR) $)^{\text {a }}$ Todos os 224 BLH do território nacional fazem parte de um sistema integrado de gestão, ou seja, integrados juntamente à rede, que permite uma organização e trabalhe em unidade de objetivos comuns. Os BLH são considerados centros de apoio ao aleitamento materno que atuam visando à Promoção, Proteção e Apoio (PPA) ao aleitamento materno, além de ser um espaço de trabalho multiprofissional ${ }^{2}$.

Em estudo anterior sobre o conhecimento e os saberes das enfermeiras do BLH acerca da assistência de Enfermagem à organização do processo de trabalho ${ }^{3}$, evidenciou-se a relevância da assistência de Enfermagem nos BLH do Estado do Espírito Santo em que o Processo de Enfermagem (PE) pode ser considerado um organizador dos trabalhos nesse espaço. Revelou-se a importância de sistematizar a assistência de Enfermagem, imbricada na observação dos saberes contidos no Manual Técnico e Normas do BLH, e, também, o diagnóstico de Enfermagem, uma vez que expressa a capacidade de os enfermeiros usarem o julgamento clínico para entender os dados coletados, possibilitando intervenções de Enfermagem para alcançar resultados positivos de saúde do binômio nas diferentes frentes de atuação profissional no BLH.

Em se tratando das ações no BLH, o trabalho da Enfermagem no cuidado direto com a PPA, inicialmente, atendia às necessidades do manejo clínico e de suas orientações, ampliando-se o seu alcance de atuação na educação em saúde, na avaliação e nos diagnósticos de Enfermagem, no planejamento das ações coletivas e na extensão do território do BLH. Dessa forma, possibilita-se a sustentabilidade de um cuidado compartilhado desde a doação do leite presente nos diversos cenários do BLH, na rota de coleta de Leite Humano Ordenhado (LHO), onde há Consulta de Enfermagem somente em espaços não hospitalares ${ }^{b}$, no ensino da técnica de coleta de LHO em domicílio e no correto armazenamento ${ }^{4,5}$.

O cuidado compartilhado corresponde a um modelo de atendimento preconizado pela Organização Mundial da Saúde (OMS) e pelo Fundo das Nações Unidas para a Infância (UNICEF) que estreita o vínculo afetivo, estimula a amamentação e envolve os pais nos cuidados com o recém-nascido. Dessa forma, considera-se uma prática importantíssima na PPA ao aleitamento materno ${ }^{6}$.

O cuidado compartilhado necessita estar alinhado com as práticas assistenciais respaldadas em conhecimentos teóricocientíficos válidos, seguindo um modelo que estimula o contato e a permanência conjunta da mãe e do bebê durante toda a internação sob os cuidados da equipe de Enfermagem. Essa prática prevê uma assistência integral e a atenção plena dos profissionais de saúde ao binômio realizadas por uma equipe multiprofissional em que as enfermeiras atuam, de forma integrada, nas ações de cuidado e orientação às famílias.

O trabalho desenvolvido no espaço do BLH exige esse compartilhamento das ações de cuidado, tendo em vista a otimização dos benefícios, tanto para a paciente quanto para a própria Enfermagem, contribuindo para o efetivo trabalho desenvolvido pela equipe que atua nesse espaço especializado, acarretando confiabilidade e segurança no processo instituído mediante a observação das normas contidas no manual técnico, bem como a execução do PE.

A proposta do trabalho em equipe pode ser considerada uma estratégia para enfrentar o intenso processo de especialização na área da saúde. Contudo, não garante, por si só, as desejadas articulação e integração das ações e dos saberes. A autora diz que "esse processo tende a aprofundar verticalmente o conhecimento e a intervenção em aspectos individualizados das necessidades de saúde" 7:103.

Nas diretrizes concernentes à Política Nacional de Atenção Básica ${ }^{8}$, o cuidado deve ser centrado na pessoa e construído com elas. As formas de coletividade são elementos relevantes para a efetivação do cuidado, muitas vezes, condicionantes ou determinantes na vida das pessoas. A coordenação do cuidado necessita de articulação mediante a gestão compartilhada da atenção integral. Por conseguinte, é relevante que os trabalhos sejam interdisciplinares e em equipe, integrando áreas técnicas e profissionais de diferentes formações. Dessa forma, defendese que seja adotada a mesma perspectiva no BLH, um conceito de trabalho em equipe sob a perspectiva da integração dos trabalhos especializados.

A temática deste estudo corresponde às ações de cuidado compartilhado em uma equipe multiprofissional de um BLH. Nas práticas de promoção, proteção e apoio ao aleitamento materno nesse espaço especializado, debruça-se sobre o cuidado, como é executado e de que forma o PE é mobilizado nesse campo. Investigam-se o eixo do cuidado e, consequentemente, a organização do trabalho profissional da enfermeira a partir do PE, ou seja: como se dão a coleta de dados, a utilização do diagnóstico de Enfermagem, o planejamento dessas ações, a execução dessas ações e sua avaliação imbricados dentro de uma equipe multiprofissional.

Ao partir da percepção, desde a aproximação com o campo mediante pesquisas ${ }^{9-11}$ realizadas, de que há diferentes formas de mobilizar as ações de cuidado, questiona-se sobre as ações autônomas exercidas pela enfermeira na atuação profissional e o processo de interlocução na equipe multiprofissional. Por 
conta do dimensionamento da organização do trabalho no BLH, os seus agentes compartilham saberes, espaços e obrigações no processo de atenção aos usuários atendidos nesse espaço especializado e setores correlacionados (maternidade, prontosocorro, neonatologia e outros).

Há uma dinâmica de trabalho com diagnósticos de Enfermagem, ações assistenciais, implementação do PE, ou seja, existe um habitus cultivado no espaço do BLH. Essas ações nesse campo revelam a disputa pelo capital simbólico e cultural desse coletivo e, nesse caso, existem modos de pensar, de agir e de operacionalizar esse conjunto de ações do PE. Assim, entende-se que "esse espaço é um espaço de lutas, uma arena onde está em jogo uma concorrência ou competição entre os agentes que ocupam as diversas posições. $\mathrm{O}$ objetivo dessas lutas reside na apropriação do capital específico do campo [...] e/ou a redefinição desse capital"12:65.

A presença ou a atuação do enfermeiro suscita reflexões sobre o entrelaçamento das ações e as normativas que existem tanto para o exercício profissional quanto para os serviços realizados nos BLH. Entende-se que o PE, de alguma forma, alcança todos os envolvidos no compartilhamento de ações de cuidado, mesmo sendo uma ação privativa da Enfermagem, uma vez que corresponde a um campo de atuação multiprofissional e revela o seu habitus ${ }^{13}$.

Desse modo, o estudo objetivou refletir sobre a organização do trabalho de Enfermagem no BLH a partir das ações de cuidado compartilhado com a equipe multiprofissional.

\section{MÉTODO}

Trata-se de uma reflexão teórica fundamentada nos conceitos de campo e habitus propostos por Pierre Bourdieu. Dessa forma o processo investigativo busca compreender as práticas sociais corriqueiras que acomodam o cotidiano e criam as estruturas simbólicas que estabelecem a ordem das coisas, das relações de poder e do habitus. Argumenta-se que o diálogo com os pressupostos teórico-metodológicos de Pierre Bourdieu permite trafegar em diversas áreas do saber com o intuito de compreender as estruturas que formam a sociedade ${ }^{14}$.

\section{RESULTADOS E DISCUSSÃO}

\section{Diálogos com Pierre Bourdieu: o campo e o habitus}

O sociólogo Pierre Bourdieu (1930-2002) é considerado um dos grandes pensadores da atualidade. Dentre os vários conceitos elaborados e estabelecidos ao longo de sua trajetória, as noções de habitus e campo possibilitam reconhecer as condicionantes dos enfermeiros nos BLH e seus desdobramentos para as políticas públicas em atenção ao aleitamento materno. Diante de uma vasta produção no campo da Sociologia, Bourdieu buscou manter uma postura de convergência dos discursos, mas, também, imprimir as suas marcas e elaborar conceitos próprios para as suas pesquisas na produção de conhecimento.
Bourdieu definiu seu conceito de campo, que ocupa um lugar central em sua Sociologia, em diálogo com diferentes universos teóricos, em particular, os de Durkheim e de Weber ${ }^{12}$ Para o sociólogo, 15:119 "os campos apresentam-se à apreensão sincrónica como espaços estruturados de posições (ou de postos) cujas propriedades dependem da sua posição nesses espaços e que podem ser analisadas independentemente das características dos seus ocupantes". Argumentou ainda que existem algumas leis gerais dos campos: são diferentes uns dos outros; possuem um funcionamento que é particular; possuem propriedades específicas; a luta está presente em qualquer campo; “Um campo define-se definindo 'paradas em jogo' e aos interesses próprios dos campos"15:120

A caracterização do BLH dialoga com o conceito proposto por Bourdieu ${ }^{13}$ no qual o social é caracterizado como microcosmos ou espaços de relações objetivas, que possuem uma lógica própria marcada por lutas em que o campo é tanto uma estrutura que constrange os agentes nele envolvidos quanto um jogo em disputa, uma vez que "Bourdieu entende o campo como um local de lutas concorrenciais que visam conservar ou transformar as relações de forças ali presentes"16:231. Nesse sentido, o campo possui uma história e uma memória que precisam ser resgatadas para compreender as ações dos seus agentes.

Conforme se constata, não se trata de uma linha contínua da história, mas um processo que envolve a construção de estratégias de enfrentamentos das dificuldades e superação de barreiras, buscando-se a qualidade do serviço e o objetivo maior, que é salvar vidas, especialmente quando os índices de mortalidade infantil demonstraram recuar com as novas práticas de manuseio do LHO. Essas transformações também foram vivenciadas nas diferentes profissões incorporadas na dinâmica de trabalho dos BLH, na especialização dos serviços e no avanço das ciências.

Dessa forma, a Enfermagem construiu um corpo teórico e prático que fundamenta suas ações de cuidado. O PE faz parte dos procedimentos de cuidado em saúde nos diferentes espaços de atuação, todavia, é importante pontuar que há uma interdependência entre os serviços prestados. Percebem-se a integralidade da proposta e a organização do trabalho do BLH a partir de uma equipe multiprofissional, adequando-se à realidade e aos contextos próprios de cada região, contando com: "médicos, nutricionistas, enfermeiros, farmacêuticos, engenheiros de alimentos, biólogos, biomédicos, médicos veterinários, psicólogos, assistentes sociais, fonoaudiólogos, terapeutas ocupacionais, auxiliares e técnicos [...], entre outros profissionais"1:23

Assim, os agentes que atuam no campo compartilham de interesses comuns e colaboram na manutenção do campo. Resulta-se, então, em uma cumplicidade objetiva que tensiona os antagonismos sobre o que faz jus ou não à luta ${ }^{15,17}$. Na teoria dos campos de Pierre Bourdieu, um campo é um microcosmo incluído no macrocosmo constituído pelo espaço social e cada campo possui regras do jogo e desafios específicos ${ }^{12: 65}$ 
Conforme constatado, "Bourdieu procura superar a oposição entre o subjetivismo e o objetivismo mediante uma relação suplementar, vertical, que medeia entre o sistema de posições objetivas e disposições subjetivas de indivíduos e coletividades"18:35. Nesse sentido, o habitus é aludido a um campo entre o sistema imperceptível das relações estruturais, que adaptam as ações e as instituições de maneira relacional pela e nas ações visíveis desses atores. "Os campos não são estruturas fixas. São produtos da história das suas posições constitutivas e das disposições que elas privilegiam"19:129.

Para Pierre Bourdieu, "os habitus são princípios geradores de práticas distintas e distintivas"13:22. Dessa forma, habitus é um conjunto de conhecimentos e disposições sociais adquiridos e incorporados ao longo do tempo que constitui uma identidade. Pode-se, então, entender por habitus o resultado das interações, perceptíveis ou não, que definem a forma de ser do indivíduo em uma determinada sociedade.

Para a Enfermagem, esse habitus está posto desde a formação inicial dos seus profissionais. Há o reconhecimento do saber próprio do campo e que as ações devem ser pautadas em conhecimentos teórico-científicos, ou seja, um conjunto de disposições sociais adquiridas e incorporadas na sua identidade. No próprio juramento de Enfermagem ${ }^{20}$, consta o compromisso de dedicar-se ao serviço da humanidade, exercer as suas funções com consciência e respeito à dignidade e aos direitos da pessoa humana, não praticar atos que coloquem em risco a integridade física ou psíquica do ser humano e exercer a Enfermagem junto à equipe de saúde, obedecendo aos preceitos da ética, da legalidade e da moral.

Portanto, trata-se de uma noção filosófica aristotélica que foi resgatada por Bourdieu na década de 1960 "para forjar uma teoria disposicional da ação capaz de reintroduzir na antropologia estruturalista a capacidade inventiva dos agentes"21:213. Na filosofia de Aristóteles, habitus significa "um estado adquirido e firmemente estabelecido do caráter moral que orienta os nossos sentimentos e desejos numa situação e, como tal, nossa conduta"21:5.

O habitus é uma noção mediadora que ajuda a romper com a dualidade de senso comum entre indivíduo e sociedade ao captar "a interiorização da exterioridade e a exteriorização da interioridade"22:47, ou seja, o modo como a sociedade se torna depositada nas pessoas sob a forma de disposições duráveis ou capacidades treinadas e propensões estruturadas para pensar, sentir e agir de modos determinados, que então as guiam nas suas respostas criativas aos constrangimentos e às solicitações do seu meio social existente.

De acordo com o sociólogo, a filosofia da ação condensada em alguns conceitos fundamentais tem, como ponto central, a relação entre as estruturas objetivas dos campos sociais e as estruturas incorporadas no habitus ${ }^{13: 10}$. Este tem como função dar conta da unidade de estilo que vincula as práticas e os bens de um agente singular ou de uma classe de agentes. Trata-se de um princípio gerador e unificador que ressignifica as características intrínsecas e relacionais de uma posição em um estilo de vida unívoco, isto é, em um conjunto unívoco de escolhas de pessoas, de bens e de práticas. Para o autor, "os habitus são princípios geradores de práticas distintas e distintivas. [...] estabelece as diferenças entre o que é bom e mau, entre o bem e o mal [...]", mantendo suas singularidades ${ }^{13: 22}$.

O conceito de habitus, em Bourdieu, é importantíssimo para a compreensão das práticas assimiladas como legítimas e ilegítimas em uma determinada sociedade e em um determinado tempo histórico. Ao eleger esse conceito para dialogar com as práticas dos enfermeiros nos BLH, especialmente a respeito do cuidado compartilhado, é importante pontuar que podem ocorrer alguns equívocos que indiquem o agente como responsável direto pelas condicionantes de trabalho nesse espaço especializado.

Nesse sentido, Bourdieu citou que a noção de habitus pode "falhar" e esclareceu que o habitus nunca é uma réplica de uma única estrutura social; não é necessariamente coerente e unificado; não está menos preparado para analisar a crise e a mudança do que está para analisar a coesão e a perpetuação e não é um mecanismo autossuficiente para a geração da ação ${ }^{21}$. De acordo com esse autor ${ }^{21}$, "[...] a convocação e o emprego dos esquemas cognitivos e motivacionais que compõem o habitus é acessível à observação metódica"21:216.

As diferentes obras do autor permitem a identificação de fundamentos desse conceito, a saber: um campo é um microcosmo incluído no macrocosmo construído pelo espaço social global, que possui regras do jogo e desafios específicos irredutíveis às regras do jogo e aos desafios dos outros campos. Constituise com um "sistema" ou um "espaço" estruturado de posições ocupadas pelos diferentes agentes do campo. Neste caso, corresponde a um espaço de lutas que reside na apropriação do capital distribuído no seio do campo desigualmente. E a cada campo corresponde um habitus (sistema de disposições incorporadas) e todo campo possui uma autonomia relativa ${ }^{12}$.

Assim, o BLH pode ser considerado um campo com todos os seus elementos constituintes. Evidencia-se que o PE, privativo do enfermeiro, coexiste com as ações do cuidado compartilhado pela equipe multiprofissional. O PE pode colaborar para equalizar as diferenças e auxiliar o enfermeiro a apropriar-se dos capitais simbólico e cultural presentes nesse campo, ampliando suas possibilidades de qualificar a atenção e o cuidado junto ao público atendido, às práticas de aleitamento materno e à assistência de Enfermagem no BLH.

Compreende-se que o PE, mesmo sendo uma ação exercida por seus profissionais, reverbera no trabalho realizado pela equipe. Nesse sentido, na execução de suas funções legais, a enfermeira mobiliza conhecimentos teórico-científicos e possui autonomia na condução do processo de atenção às usuárias no BLH, no diagnóstico e na prescrição de Enfermagem. Contudo, essas usuárias também são atendidas por outros profissionais da equipe do BLH de acordo com a necessidade de cada caso. Em se tratando do LHO, existe uma cadeia de ações que convida que sua execução contemple um trabalho colaborativo e cooperativo até a entrega final do seu produto, adequado e próprio para o consumo. Percebe-se, ainda, que a coleta de dados para fins de atendimento e acompanhamento 
das usuárias é uma ação que permite a condução e a tomada de decisões junto à equipe do BLH.

O processo de trabalho de uma equipe multiprofissional está organizado a partir das ações de cuidado compartilhadas pela Enfermagem nos BLH e vinculadas a um hospital/maternidade com atenção maternoinfantil. Infere-se que há uma organização e uma divisão do trabalho. Cada profissional sabe quais são suas funções e exerce-as tanto rotineiramente, ou seja, há uma rotina de trabalho a cumprir, quanto por demanda. Percebe-se uma estrutura gerencial do cuidado em que a paciente, ao dar entrada no hospital ou no BLH, conta com uma equipe que busca uma resposta àquela questão de saúde até encontrar uma solução definitiva ou encaminhamentos necessários. $O$ trabalho em equipe fica nítido quando se efetivam a comunicação e a acessibilidade ou quando as vias de acionamento do cuidado necessário ao binômio estão incorporadas como um habitus nesse campo.

Dessa forma, é possível manter esse diálogo entre as condicionantes sociais que regem a utilização do $P E$ nos espaços de atuação do enfermeiro conforme a Resolução COFEN nº $358 / 2009$ e as ações que compõem os habitus desses sujeitos. Essa perspectiva é corroborada por Pontes ${ }^{23}$ ao utilizar o referencial teórico de Pierre Bourdieu 13,17,19,22 para analisar as ações e a participação da Enfermagem na consolidação do espaço do BLH em uma universidade pública do Espírito Santo.

Diante disso, as ações da Enfermagem estão estruturadas mediante o processo histórico estruturante dos habitus presentes no campo. O cuidado é considerado uma das principais ações da Enfermagem ${ }^{24,25}$. Tem fortalecido a construção do saber da área, sendo a essência da profissão, a base do ensino e da prática de Enfermagem. Percebe-se que há mudanças de habitus e campo com a integralização do cuidado compartilhando entre os profissionais de saúde ${ }^{26}$.

Um habitus incorporado pela equipe do BLHé a capacitação para o serviço como uma necessidade constante. Esse movimento exige a articulação da equipe para fomentar esse processo de educação continuada. No caso, cada profissional pode colaborar com sua especialidade e experiência no BLH. Tanto a Enfermagem é convidada a organizar e a ministrar cursos e capacitação quanto os outros profissionais exercem essa função de organizar momentos de troca de conhecimento na equipe. Quanto mais capacitada e entrosada for a equipe, melhores serão a articulação do serviço e a atenção ao cuidado com o binômio no BLH e demais setores.

\section{CONCLUSÃO E IMPLICAÇÕES PARA A PRÁTICA}

O cuidado compartilhado é reconhecido como importante fator de equalização da assistência e demais serviços realizados no BLH. Há uma parceria e cumplicidade nas ações desenvolvidas em que cada profissional sabe, exatamente, qual é a sua função, sua responsabilidade e em qual momento ou demanda faz-se necessária a sua presença. A avaliação clínica de cada caso exige a consciência ética e profissional dos agentes para oferecer o melhor tratamento possível no BLH. Há o habitus da distribuição do trabalho, que segue um fluxo incorporado na dinâmica de atuação em equipe. A estratégia é manter a comunicação aberta e, também, manter a predisposição para atender a eventos que surgem na rotina ou emergências que chegam ao BLH.

Ao utilizar os conceitos de Pierre Bourdieu, remetendo-os para o cuidado compartilhado, é possível inferir o habitus nos modos de cuidar, considerando-se, ainda, aquele específico construído ao longo da história da Enfermagem. Nesse caso, o PE pode ser tomado como um "princípio gerador e unificador do conjunto das práticas e das ideologias características de um grupo de agentes"27:191, ou seja, a Enfermagem e seus habitus no campo do BLH.

Eis o grande desafio posto para o cuidado compartilhado no BLH: convergir as ações de cuidado respeitando e valorizando os saberes e fazeres próprios de cada profissional que compõe a equipe; mobilizar os conhecimentos em prol da oferta de um serviço público de qualidade e comprometido com a promoção, proteção e apoio ao aleitamento materno; investir na formação continuada para otimizar as ações de cuidado ao binômio nos diferentes espaços de atuação da equipe do BLH e continuar a colaborar com a redução da mortalidade infantil a partir da oferta de leite humano, com controle e qualidade no seu processo realizado por várias mãos.

A limitação deste estudo consiste na dificuldade em operar com outros conceitos de Pierre Bourdieu, especialmente, a noção de capital cultural e capital simbólico, que mantêm estreita relação com o campo e o habitus. Novas reflexões são requeridas para compreender as nuances do campo científico, do espaço social e da dominação que atravessam o campo das políticas públicas em saúde, sobretudo quanto ao aleitamento materno e ao BLH.

\section{CONTRIBUIÇÕES DOS AUTORES}

Desenho do estudo de reflexão. Giovanna Rosario Soanno Marchiori. Valdecyr Herdy Alves. Diego Pereira Rodrigues. Bianca Dargam Gomes Vieira. Audrey Vidal Pereira. Tatiana do Socorro dos Santos Calandrini.

Análise de dados. Giovanna Rosario Soanno Marchiori. Valdecyr Herdy Alves. Diego Pereira Rodrigues. Bianca Dargam Gomes Vieira. Audrey Vidal Pereira. Tatiana do Socorro dos Santos Calandrini.

Interpretação dos resultados. Giovanna Rosario Soanno Marchiori. Valdecyr Herdy Alves. Diego Pereira Rodrigues. Bianca Dargam Gomes Vieira. Audrey Vidal Pereira. Tatiana do Socorro dos Santos Calandrini.

Redação e revisão crítica do manuscrito. Giovanna Rosario Soanno Marchiori. Valdecyr Herdy Alves. Diego Pereira Rodrigues. Bianca Dargam Gomes Vieira. Audrey Vidal Pereira. Tatiana do Socorro dos Santos Calandrini.

Aprovação da versão final do artigo. Giovanna Rosario Soanno Marchiori. Valdecyr Herdy Alves. Diego Pereira Rodrigues. Bianca Dargam Gomes Vieira. Audrey Vidal Pereira. Tatiana do Socorro dos Santos Calandrini. 
Responsabilidade por todos os aspectos do conteúdo e a integridade do artigo publicado. Giovanna Rosario Soanno Marchiori. Valdecyr Herdy Alves. Diego Pereira Rodrigues. Bianca Dargam Gomes Vieira. Audrey Vidal Pereira. Tatiana do Socorro dos Santos Calandrini.

\section{EDITOR ASSOCIADO}

Stela Maris de Melo Padoin (1)

\section{EDITOR CIENTÍFICO}

\author{
Ivone Evangelista Cabral (D)
}

\section{REFERÊNCIAS}

1. Agência Nacional de Vigilância Sanitária. Banco de leite humano: funcionamento, prevenção e controle de riscos/Agência Nacional de Vigilância Sanitária. Brasília: ANVISA; 2008. 160 p.

2. Barros M, Almeida J. (2019). Rede Brasileira de Bancos de Leite Humano: uma rede baseada na confiança. Pesq Bras Ciênc In Biblioteconomia (Online). 2019;14(1):125-33. https://doi.org/10.22478 ufpb.1981-0695.2019v14n1.45180.

3. Marchiori GRS. Sistematização da Assistência de Enfermagem nos Bancos de Leite Humano do Estado do Espírito Santo: saberes dos Enfermeiros. Rio de Janeiro: UFF/Faculdade de Medicina; 2015.

4. Portaria $n . .698 / G M$, de 09 de abril de 2002 (BR). Define a estrutura e as normas de atuação e funcionamento dos Bancos de Leite Humano - BLH. Diário Oficial da União [periódico na internet], Brasília (DF), 9 abril 2002 [citado 2020 jan 11]. Disponível em: http://www.redeBLH. fiocruz.br/media/698 [1].pdf

5. Hospital Israelita Albert Einstein. Cuidado Compartilhado [Internet] 2021 [citado 2021 maio 10]. Disponível em: https://www.einstein.br/ estrutura/maternidade/alojamento-conjunto

6. Resolução COFEN n॰ 358 de 15 de outubro de 2009 (BR). Dispõe sobre a Sistematização da Assistência de Enfermagem - SAE - nas Instituições de Saúde Brasileiras. Diário Oficial da União [periódico na internet], Brasília (DF), 2009 [citado 2021 maio 10]. Disponível em: http://www.cofen.gov.br/resoluo-cofen-3582009_4384.html

7. Peduzzi M. Equipe multiprofissional de saúde: conceito e tipologia. Rev Saude Publica. 2001;35(1):103-9. http://dx.doi.org/10.1590/S003489102001000100016. PMid:11285526.

8. Portaria $n^{\circ} 2.488$, de 21 de outubro de 2011 (BR). Política Nacional de Atenção Básica. Diário Oficial da União, Brasília (DF), 2011.

9. Marchiori GRS, Alves VH, Rodrigues DP, Gabriel AD, Santos MV, Branco MBLR. Articulação entre sistematização de Enfermagem e procedimentos operacionais: perspectiva dos enfermeiros dos bancos de leite. Rev Enferm UFSM. 2017 abr/jun;7(2):1-14. http://dx.doi. org/10.5902/2179769225701.
10. Marchiori GRS, Alves VH, Rodrigues DP, Santos MV, Branco MBLR, Gabriel AD. Saberes sobre processo de Enfermagem no banco de leite humano. Texto Contexto Enferm. 2018;27(2):e0390016. http://dx.doi. org/10.1590/0104-070720180000390016.

11. Marchiori GRS, Alves VH, Pereira AV, Vieira BDG, Rodrigues DP, Dulfe PM, et al. Nursing actions in human milk banks in times of COVID-19. Rev Bras Enferm. 2020;73(Suppl 2):e20200381. https:// doi.org/10.1590/0034-7167-2020-0381.

12. Lahire BC. Campo. In: Catani AM, Nogueira MA, Hey AP, Medeiros CCC, organizadores. Vocabulário Bourdieu. Belo Horizonte: Autêntica, 2017. p. 64-66.

13. Bourdieu P. Razões práticas: sobre a teoria da ação. Campinas, SP Papirus; 1996.

14. Guimarães VOS. Análise do Método em Pierre Bourdieu. Rev Cient Educ. [Internet]. 2018 [citado 2021 maio 10];3:e019008. Disponível em: https://seer.facmais.edu.br/rc/index.php/RCE/article/view/50

15. Bourdieu P. Algumas propriedades dos campos. In. Bourdieu P. Questões de Sociologia. Fim de Século. Lisboa: Edições Sociedade Unipessoal; 2003.

16. Brandão Z. Operando com conceitos: com e para além de Bourdieu. Educação e Pesquisa, São Paulo. 2010 jan/abr;36(1):227-41. http:// dx.doi.org/10.1590/S1517-97022010000100003.

17. Bourdieu P. O poder simbólico. Lisboa: Difel: 1989.

18. Thiry-Cherques HR. Pierre Bourdieu: a teoria na prática. Rev Adm Pública (Online). 2006;40(1):27-56. http://dx.doi.org/10.1590/S003476122006000100003.

19. Bourdieu P. Meditações pascalianas. Rio de Janeiro: Bertrand Brasil, 2001.

20. Resolução Cofen-218/1999 (BR). Aprova o Regulamento que disciplina sobre Juramento, Símbolo, Cores e Pedra utilizados na Enfermagem. Diário Oficial da União, Brasília (DF), 1999.

21. Wacquant L. Habitus. In: Catani AM, Nogueira MA, Hey AP, Medeiros CCC, organizadores. Vocabulário Bourdieu. Belo Horizonte: Autêntica; 2017.

22. Bourdieu P. Sociologia. São Paulo: Ática, 1983.

23. Pontes MB, Santos TCF, Nogueira ALL, Peres MAA, Rios MZ, Almeida Fo AJ. Human milk bank: challenges and visibility for nursing. Texto contexto - enferm. 2017;26(2):e3760015. http://dx.doi.org/10.1590/010407072017003760015.

24. Bueno FMG, Queiroz MS. O enfermeiro e a construção da autonomia profissional no processo de cuidar. Rev Bras Enferm. 2006;59(2):222-7. https://doi.org/10.1590/S0034-71672006000200019.

25. Ferreira MA. Enfermagem: arte e ciência do cuidado. Esc Anna Nery. 2011;15(4):664-6. http://dx.doi.org/10.1590/S1414-81452011000400001.

26. Espirito Santo FH, Porto IS. De Florence Nightingale às perspectivas atuais sobre o cuidado de Enfermagem: a evolução de um saber/fazer. Esc Anna Nery, 2006;10(3):539-46. https://doi.org/10.1590/S141481452006000300025.

27. Bourdieu P. A economia das trocas simbólicas. São Paulo: Perspectiva, 2007.

\footnotetext{
${ }^{a} \mathrm{~A}$ Rede Brasileira de Bancos de Leite Humano (rBLH-BR) configura-se como ação estratégica da Política Nacional de Aleitamento Materno e, além de coletar processar e distribuir leite humano a bebês prematuros e de baixo peso, os Bancos de Leite Humano (BLH) realizam atendimento de orientação e apoio à amamentação. [...] O modelo brasileiro é reconhecido mundialmente pelo desenvolvimento tecnológico inédito que alia baixo custo à alta qualidade, além de distribuir o leite humano conforme as necessidades específicas de cada bebê, aumentando a eficácia da iniciativa para a redução da mortalidade neonatal (Fonte: https://rblh.fiocruz.br/quem-somos).
}

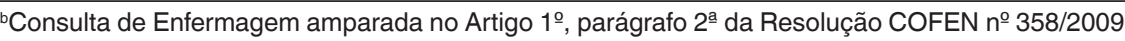

